\title{
Long-term efficacy and duration of action of dexamethasone implant, in vitrectomised and non-vitrectomised eyes with persistent diabetic macular oedema
}

\author{
George G. Bastakis $\mathbb{B D}^{1} \cdot$ Dimitris Dimopoulos ${ }^{1} \cdot$ Anastasios Stavrakakis $^{1} \cdot$ George Pappas $^{1}$
}

Received: 20 January 2018 / Revised: 4 August 2018 / Accepted: 16 August 2018 / Published online: 9 October 2018

(c) The Royal College of Ophthalmologists 2018

\begin{abstract}
Purpose To evaluate the efficacy and duration of action of an intravitreal (dexamethasone (Ozurdex)) implant in vitrectomised and non-vitrectomised eyes with persistent diabetic macular oedema (DMO).

Methods We retrospectively analysed the records for 18 eyes that had or had not been vitrectomised but required an intravitreal dexamethasone implant for DMO after a poor response to anti-vascular endothelial growth factor. Optical coherence tomography and visual acuity (VA) examinations were performed before and 1, 3 and 6 months after implantation. The six months following implantation constituted one treatment round; up to three rounds were studied. Results Ten of 18 eyes had undergone vitrectomy. Best-corrected visual acuity (BCVA) and central macular thickness (CMT) were significantly improved by months $1-3$ after implantation of the Ozurdex device in all rounds of treatment. The BCVA and CMT deteriorated gradually after month 3 through to month 6 post implantation. There were no statistically significant differences between the vitrectomised and non-vitrectomised groups at any time point. When the implantation interval was $<6$ weeks from the end of each treatment round, the improvement in BCVA and CMT was obvious even after 18 months of treatment.

Conclusions Vitrectomy did not have a negative effect on the duration of action or efficacy of the Ozurdex implant in patients with persistent DMO. The implant started working from the first month after implantation regardless of whether vitrectomy had or had not been performed. The maximum functional and anatomic improvement was achieved in the first 3 months post implantation in all treatment rounds.
\end{abstract}

\section{Introduction}

Macular oedema is characterised by accumulation of extracellular fluid between the retinal (inner nuclear and outer plexiform) layers, forming extracellular oedema, or inside the cells, forming intracellular oedema (Muller cells) $[1,2]$. Therefore, macular oedema can be categorised as cytotoxic (intracellular) or vasogenic

Electronic supplementary material The online version of this article (https://doi.org/10.1038/s41433-018-0219-8) contains supplementary material, which is available to authorized users.

George Pappas

gpappasresearch@gmail.com

retinacrete@gmail.com

1 Ophthalmology Clinic, Medical Retina \& Vitreoretinal Surgery Department, Venizeleio Hospital of Crete, Knossos avenue 44, Crete, 71409, Heraklion, Greece (extracellular). Vasogenic macular oedema (MO) develops as a result of breakdown of the blood-retina barrier and inability of the cellular mechanisms (neuronal and glial) in the retina to decompensate and restore the integrity of the layers to normal [3]. MO is a common pathological endpoint of many pathological ocular entities, including diabetic retinopathies, retinal vein occlusion, retinal surface pathologies and inflammatory conditions (uveitis or inflammation after ocular surgery) $[2,4]$. Deterioration of central visual acuity is common to all of the above conditions, making MO a highly troublesome pathology for the patient and a therapeutic challenge for the ophthalmological community.

In the majority of these pathologies, a specific repertoire of inflammatory mediators and molecular factors contribute to the damage that occurs at the blood-retina barrier and subsequently to formation of MO. These molecules include vascular endothelial growth factor (VEGF), angiotensin-II, cytokines, prostaglandins, 
interleukins and angiopoietin-2 [5-8]. Anti-angiogenic factors (mainly anti-VEGFs) and anti-inflammatory factors (corticosteroids) have been used successfully for the treatment of MO. In addition to their anti-inflammatory activity, corticosteroids also have anti-angiogenic and anti-permeability properties that make them useful drugs in the treatment of MO. Unfortunately, oral corticosteroids are known to have a broad range of systemic side effects [9-11]. In contrast, intravitreal steroids reduce the risk of systemic side effects and increase the availability and concentration of the drug in the area where it is mostly needed $[12,13]$. Protocol I in the DRCR study showed that use of intravitreal triamcinolone combined with laser therapy was as effective as ranibizumab combined with laser therapy for improving best-corrected visual acuity (BCVA) and decreasing central retina thickness. The improvement in BCVA lasted up until 24 weeks and was gradually reduced thereafter because of formation of cataracts. In the same study, triamcinolone acetonide combined with laser was found to be as effective as ranibizumab alone and superior to laser monotherapy in a pseudophakic subgroup [14-16]. Moreover, previous studies had shown that a 4-mg dose of triamcinolone acetonide could be detected inside the human vitreous for three months post injection [17].

The introduction of implants that slowly release steroids (dexamethasone, fluocinolone) has made it possible to extend drug availability in the vitreous in therapeutic concentrations [18, 19]. An intravitreal dexamethasone implant (Ozurdex; Allergan, Inc., Irvine, CA, USA) has been approved by the US Food and Drug Administration for the treatment of diabetic macular oedema (DMO) [18, 20]. The major side effects of intravitreal steroid implants are development and progression of cataract in phakic eyes and increased intraocular pressure (IOP) [21]. In severe cases, both of these complications can lead to a need for incisional glaucoma surgery for regulation of IOP or cataract surgery.

None of the patients who participated in the above studies had undergone vitrectomy. The efficacy of intravitreal steroid implants in vitrectomised eyes is questionable given the increased rates of clearance from the vitreous cavity found with many drugs. This increase in drug clearance results in a shorter duration of exposure of the retinal tissue to the drug, thereby decreasing its efficacy [22]. Anti-VEGF agents and corticosteroids (e.g. triamcinolone) along with other intravitreal drugs (e.g. 5-fluorouracil and amphotericin) show the previously described pharmacokinetic profile in vitrectomised eyes [22-26]. In the CHAMPLAIN study, a single dexamethasone implant improved vision and central macular thickness (CMT) when compared with baseline values. All patients in the CHAMPLAIN study had difficult-to-treat DMO and had undergone vitrectomy in the past [27]. The results of our present study support the assumption that the efficacy of the dexamethasone intravitreal implant is not affected by previous vitrectomy surgeries in eyes with persistent DMO during 18 months of treatment. There was no significant difference in the timing of initiation of effect nor the duration of action of the implant between the vitrectomised group and the nonvitrectomised group.

\section{Methods}

\section{Enrolment of patients and examination}

We retrospectively analysed the medical records for 17 consecutive patients (18 eyes) who required an intravitreal dexamethasone implant for DMO. Ten eyes had undergone 3-port 23-gauge pars plana vitrectomy in the past for epiretinal membrane peeling, dense vitreous haemorrhage, or advanced proliferative diabetic retinopathy (the vitrectomy group). Eight eyes had no history of posterior or anterior vitrectomy (the non-vitrectomy group). All patients in each group had undergone monthly treatment with anti-VEGF agents and a corticosteroid (triamcinolone) for DMO in the past. None of the patients in either group responded to the anti-VEGF injections. Non-responsive/persistent DMO was defined as DMO with a CMT $>280 \mu \mathrm{m}$ or with a CMT that never decreased more than $40 \%$ from baseline values, despite treatment with anti-VEGF and triamcinolone for at least 6 months. Furthermore, all patients had previously received at least 1 triamcinolone injection (range 1-3) and showed more than a 30\% reduction in CMT after 2 months or a reduction $\geq 100 \mu \mathrm{m}$ from baseline (see Table 1). The exclusion criteria in both groups were uncontrolled glaucoma, history of infectious uveitis, a known steroid-related increase in IOP (steroid responders), permanent macular damage, and anti-VEGF, triamcinolone, or laser therapy in the 3 months before starting treatment with the Ozurdex implant. OCT was performed and BCVA measurements were obtained in all patients before and 1, 3 and 6 months post implantation. BCVA was measured using Snellen charts and converted to logarithm of the minimum angle of resolution $(\log M A R)$ units for the statistical analysis. One treatment round consisted of the 6 months post implantation. A maximum of 3 treatment rounds (18 months) was studied. Seven patients in the vitrectomised group completed the 3 rounds of treatment, 1 completed 2 rounds and two completed only 1 round. Six patients (7 eyes) in the non-vitrectomised group completed the 3 rounds of treatment and 1 patient completed only 1 round. The interval between month 6 and the next implant was always less than 6 weeks in the patients who received more than one implant. The patient demographics and clinical 
Table 1 General clinical characteristics of patients

\begin{tabular}{lll}
\hline & Vitrectomised group & Non-vitrectomised group \\
\hline Phacic/pseudophakic & $80 \%$ pseudophakic & $62 \%$ pseudophakic \\
Mean number of a-VEGF inj. & 4.5 & 4.7 \\
Mean number of triamcinolone inj. & 1.9 & 1.3 \\
Mean number of Ozurdex & 2.5 & 2.7 \\
Males/females & $5 \mathrm{M} / 5 \mathrm{~F}$ & $3 \mathrm{M} / 4 \mathrm{~F}$ \\
$\begin{array}{l}\text { Mean Age } \\
\text { Mean BCVA improvement (logMar) between baseline- }\end{array}$ & 0.39 & 76 \\
$\begin{array}{l}\text { Mean BCVA improvement (logMar) between baseline- } \\
\text { Menths }\end{array}$ & 0.39 & 0.28 \\
$\begin{array}{l}\text { Men months } \\
\text { Mean BCVA improvement (logMar) between baseline- }\end{array}$ & 0.22 & 0.26 \\
$\begin{array}{l}\text { l8 months } \\
\text { Mean CMT reduction in } \mu \mathrm{m}(\%) \text { between baseline-13 } \\
\text { months }\end{array}$ & $256(47 \%)$ & 0.14 \\
$\begin{array}{l}\text { Mean CMT reduction in } \mu \mathrm{m}(\%) \text { between baseline-15 } \\
\text { months }\end{array}$ & $239(44 \%)$ & $350(54 \%)$ \\
$\begin{array}{l}\text { Mean CMT reduction in } \mu \mathrm{m}(\%) \text { between baseline-18 } \\
\text { months }\end{array}$ & $174(32 \%)$ & $329(51 \%)$ \\
\hline
\end{tabular}

characteristics are shown in Table 1. All patients underwent fluorescein angiography before receiving their first implant. Measurement of IOP, slit-lamp examination and indirect ophthalmoscopy were also performed in all patients. No serious adverse events, such as endophthalmitis, vitreous haemorrhage, implant migration into the anterior chamber or uncontrolled glaucoma needing surgical intervention, occurred in our study. All adverse events are shown in Table 2. Informed consent was obtained from all patients enrolled in this study. The study and data accumulation were carried out with the approval of the Institutional Review Board/ethics committee (Venizeleio hospital Scientific council, \#12935, decision:67/15).

\section{Surgical procedures}

Three-port 23-gauge pars plana vitrectomy was performed in all patients in the vitrectomy group by the same surgeon (GP) using the same setting. Following removal of the epiretinal membrane, the internal limiting membrane was peeled in all patients. Epiretinal membrane peeling was performed without any dye assistance. Brilliant Blue-G dye was used to stain the internal limiting membrane.

\section{Statistical analysis}

The normality of the data were tested using the D'Agostino $\&$ Pearson omnibus test. The paired two-tailed Student's $t$ test was used to test changes in BCVA and CMT in each study group at different time points for statistical significance. Two-way analysis of variance (ANOVA) was used to compare differences in BCVA and CMT between
Table 2 Intraocular pressure analysis and adverse events in the study eyes

\begin{tabular}{|c|c|c|}
\hline & $\begin{array}{l}\text { Vitrectomised } \\
\text { group }\end{array}$ & $\begin{array}{l}\text { Non- } \\
\text { vitrectomised } \\
\text { group }\end{array}$ \\
\hline $\begin{array}{l}\text { No of patients with } \mathrm{IOP}>20 \\
\mathrm{mmHg}(\%)\end{array}$ & $5(50 \%)$ & $2(29 \%)$ \\
\hline $\begin{array}{l}\text { No of patients with } \mathrm{IOP}>24 \\
\mathrm{mmHg}(\%)\end{array}$ & $3(30 \%)$ & $1(14 \%)$ \\
\hline \multicolumn{3}{|c|}{ Patients first presentation of IOP $>20 \mathrm{mmHg} /$ treatment round (\%) } \\
\hline 1st round & $\begin{array}{l}3 \text { patients } \\
(30 \%)\end{array}$ & 1 patient $(14 \%)$ \\
\hline 2nd round & 1 patient $(10 \%)$ & 1 patient $(14 \%)$ \\
\hline 3rd round & 1 patient $(10 \%)$ & None \\
\hline \multicolumn{3}{|l|}{ Mean IOP per treatment round } \\
\hline Baseline & $12.9 \mathrm{mmHg}$ & $13.5 \mathrm{mmHg}$ \\
\hline End of 1 st round & $14.2 \mathrm{mmHg}$ & $14.2 \mathrm{mmHg}$ \\
\hline End of 2nd round & $15.4 \mathrm{mmHg}$ & $15.1 \mathrm{mmHg}$ \\
\hline End of 3 rd round (18 months) & $15.0 \mathrm{mmHg}$ & $15.1 \mathrm{mmHg}$ \\
\hline $\begin{array}{l}\text { Mean IOP increase in } 18 \text { months } \\
\text { of treatment }\end{array}$ & $+2.1 \mathrm{mmHg}$ & $+1.67 \mathrm{mmHg}$ \\
\hline \multicolumn{3}{|l|}{ Other adverse events } \\
\hline Conjunctiva haemorrhage $(\%)$ & $4(40 \%)$ & $3(42.8 \%)$ \\
\hline Mild ocular pain (\%) & $2(20 \%)$ & $3(42.8 \%)$ \\
\hline Foreign body sensation (\%) & $2(20 \%)$ & $2(28.5 \%)$ \\
\hline
\end{tabular}

the vitrectomised and non-vitrectomised groups at the same time points. The data are shown as the mean and standard deviation. Intragroup differences in BCVA and CMT between the different time points from baseline, the second 
implant and the third implant were tested using one-way ANOVA with the Geisser-Greenhouse correction and Dunnett multiple comparisons test. A $p$-value $<0.05$ was considered statistically significant. The confidence interval was $95 \%$. The statistical analysis was performed using GraphPad Prism version 6.0 for Windows (GraphPad Software Inc., La Jolla, CA, USA, www.graphpad.com).

\section{Results}

We analysed the BCVA and CMT at different time points to compare the efficacy and duration of action of the dexamethasone implant between our vitrectomised group and our non-vitrectomised group. The pattern of changes in BCVA post implantation indicated rapid improvement during the first month that was sustained for at least 3 months. Thereafter, there was a gradual decrease in BCVA; however, the final value at 6 months post implantation was better than the baseline value in both groups. The BCVA followed the same pattern after the second (7-12 months) and third (13-18 months) dexamethasone implants in both study groups (Fig. 1a).

After the first round of treatment, the mean BCVA improved from $0.64 \pm 0.36$ at baseline to $0.36 \pm 0.17,0.36$ \pm 0.18 and $0.43 \pm 0.20$ after 1,3 and 6 months, respectively, in the vitrectomised group (Fig. 2a). The mean difference from the baseline value of 0.28 was statistically significant at 1 and 3 months $(p=0.0172$ and $p=0.0084$, respectively). Six months post implantation, the mean difference from baseline was 0.21 . In the non-vitrectomised group, the mean BCVA increased from $0.60 \pm 0.20$ at baseline to 0.31 \pm 0.14 and $0.31 \pm 0.15$ after 1 and 3 months, respectively. Six months post implantation, we observed a decrease in BCVA of $0.42 \pm 0.15$. Like in the vitrectomised group, the mean difference between the baseline BCVA was 0.29 at month 1 and 0.28 at month 3 ; both values were statistically significant $(p=0.0008$ and $\mathrm{p}=0.0027$, respectively). The mean difference in BCVA between baseline and 6 months post implantation was 0.17 in the non-vitrectomised group and was statistically significant (Fig. 2a).

The mean BCVA was $0.47 \pm 0.11$ in the vitrectomised group before the second dexamethasone implant. The mean VA at that time was improved by two lines from baseline but was slightly worse than the mean value at 6 months. The mean BCVA was $0.25 \pm 0.17$ at 1 month after the second implant and was $0.26 \pm 0.17$ after a further 3 months. Six months after the second implant, the mean BCVA was 0.36 \pm 0.11 . The mean differences in BCVA values before the second implant and those at 7,9 and 12 months were all statistically significant $(p=0.0025, p=0.0045$ and $p=$ 0.0117 , respectively; Fig. 2b). In the non-vitrectomised group, the mean BCVA was $0.47 \pm 0.13$ before the second
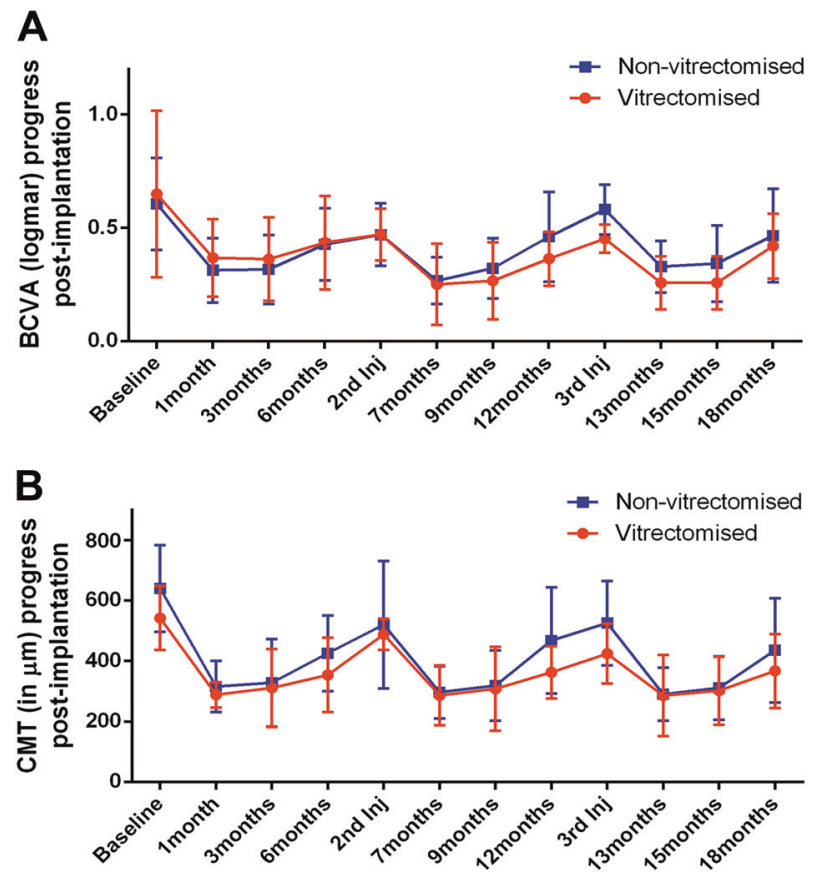

Fig. 1 Changes in best-corrected visual acuity (BCVA) and central macular thickness (CMT) during three rounds of treatment (lasting 18 months) in vitrectomised and non-vitrectomised groups. a Graph showing the changes in BCVA from baseline until the end of 18 months of follow-up. b Graph showing the changes in CMT $(\mu \mathrm{m})$ from baseline until the end of 18 months of follow-up. The baseline, second implant and third implant entries represent the starting points of three different rounds of treatment (first to third implants). The red lines indicate the vitrectomized group. The blue lines indicate the nonvitrectomized group. The data are shown as the mean. The error bars show the standard deviation

dexamethasone implant. In this group, like in the vitrectomised group, the mean BCVA at this point was improved from baseline by 1.5 lines but was slightly worse than the mean VA at 6 months. After the second implant, the mean BCVA was $0.26 \pm 0.10$ at 1 month, $0.32 \pm 0.13$ at 3 months and $0.46 \pm 0.18$ at 6 months. The mean difference in the BCVA value before the second implant was statistically significant at 1 month $(0.20 ; p=0.0072)$ and 3 months $(0.15, p=0.0209)$. In the non-vitrectomised group, there was no significant difference between the BCVA value before and 6 months after the second implant (12 months; Fig. 2b).

Finally, the third round of treatment in the vitrectomised group achieved an improved mean BCVA $(0.45 \pm 0.06,2$ lines) before the implant when compared with the baseline value. The mean BCVA values at 1 and 3 months after initiation of the third round were $0.25 \pm 0.13$ and $0.25 \pm$ 0.11 , respectively. However, the difference from the preimplantation VA was 0.20 for both time points and statistically significant $(p=0.003$ and $p=0.0082)$. On this round, the mean BCVA at 6 months was $0.41 \pm 0.14$ with not significantly different from the pre-implantation BCVA. 

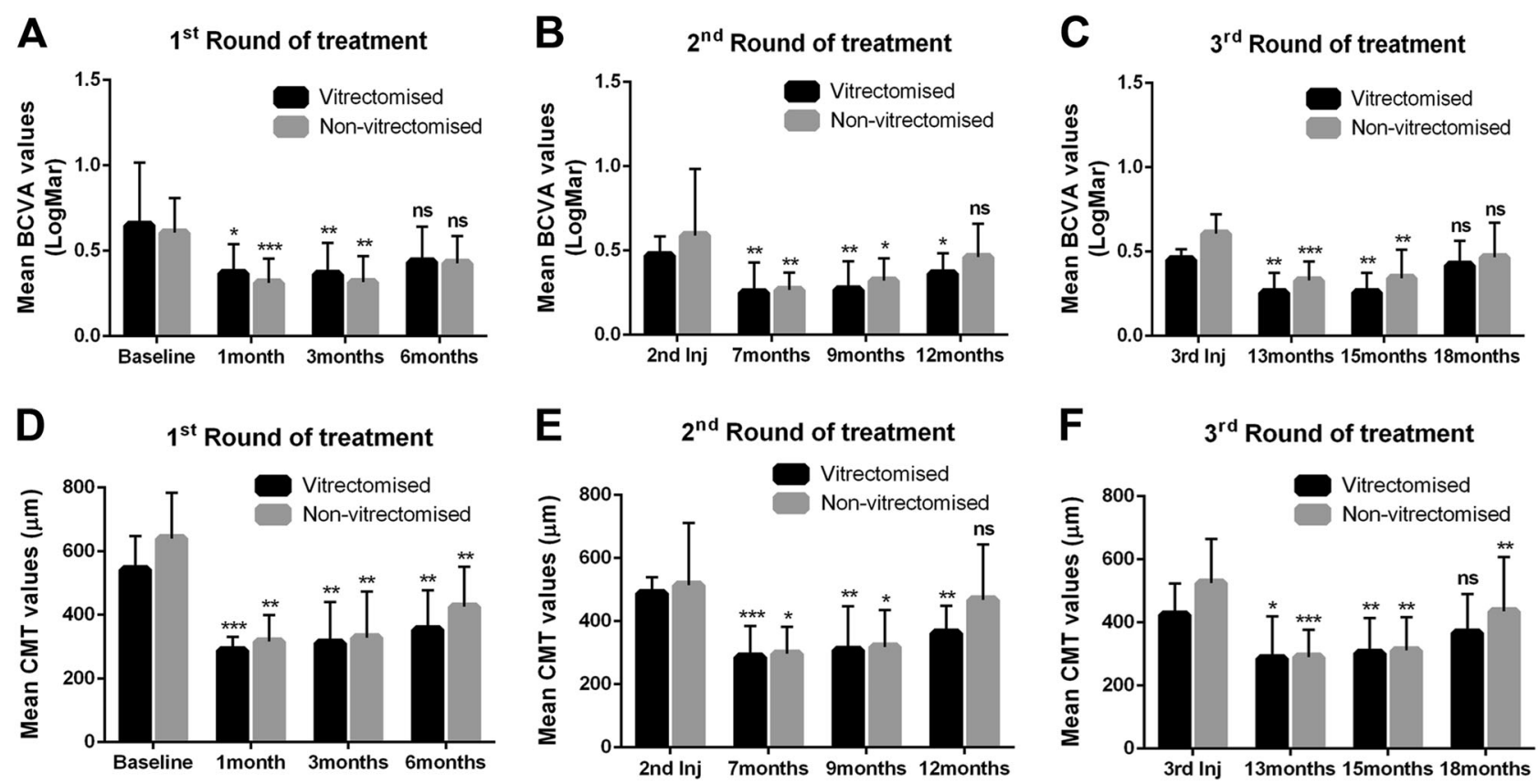

Fig. 2 Mean best-corrected visual acuity (BCVA) and central macular thickness (CMT) in vitrectomised and non-vitrectomised groups during three rounds of treatment. a Differences in BCVA during round 1 of treatment in both groups. b Differences in BCVA during round 2 of treatment in both groups. c Differences in BCVA during round 3 of treatment in both groups. d-f Quantification of mean CMT during

rounds 1, 2 and 3 of treatment. The data are shown as the mean. The error bars show the standard deviation. Significant difference from baseline, second implant and third implant time points presented with $* p<0.05, * * p<0.01, * * * p<0.001$, ns no statistically significant difference

In the non-vitrectomised group, the mean BCVA was 0.58 \pm 0.11 before the third implant, $0.32 \pm 0.11$ after month 1 , $0.34 \pm 0.16$ after month 3 and $0.46 \pm 0.2$ at month 6 post implantation. The mean difference between the pretreatment and post treatment BCVA was $0.25,0.23$ and 0.11 , respectively after 1,3 and 6 months of treatment. The difference was statistically significant only for months 1 ( $p=0.0008)$ and 3 ( $\mathrm{p}=0.008$; Fig. 2c).

The CMT in both groups responded in a similar temporal manner as the BCVA. A rapid decrease was observed in month 1, and this improvement persisted for 3 months post implantation. After month 3, there was a gradual increase in CMT until month 6 (Fig. 1b).

For the first round of treatment, the mean CMT in the vitrectomised group was $542 \mu \mathrm{m} \pm 105$ at baseline and decreased to $288 \pm 42$ and $311 \pm 128 \mu \mathrm{m}$, respectively, by months 1 and 3 post implantation. Six months later, the CMT increased to $353 \pm 123 \mu \mathrm{m}$ as the potency of the implant slowly declined (Fig. 2d). The mean difference between the baseline CMT and that 1 month post implantation was statistically significant $(272 \mu \mathrm{m} ; p=0.0003)$, as it was at 3 months $(226 \mu \mathrm{m}, p=0.0026)$ and 6 months (184 $\mu \mathrm{m}, \quad p=0.0056)$ post implantation. In the nonvitrectomised group, the baseline CMT of $640 \pm 143 \mu \mathrm{m}$ decreased to $315 \pm 84 \mu \mathrm{m}$ at 1 month and was $328 \pm 144 \mu \mathrm{m}$ at 3 months. The final CMT at 6 months post implantation was $425 \pm 125 \mu \mathrm{m}$ (Fig. 2d). The mean difference between

the CMT at baseline and that at the different time points was statistically significant for months $1(324 \mu \mathrm{m}, p=0.0023), 3$ $(312 \mu \mathrm{m}, p=0.0049)$ and $6(214 \mu \mathrm{m}, p=0.0065)$ post implantation.

During the second round of treatment, the mean CMT value before the implant was $488 \pm 51 \mu \mathrm{m}$ in the vitrectomised group, which was worse than the CMT at 6 months but was improved related to the baseline CMT. The mean CMT values at months 1,3 and 6 post implantation were $286 \pm 98,308 \pm 138$ and $362 \pm 86 \mu \mathrm{m}$, respectively. Moreover, the mean difference in CMT between before the second implant and 1, 3 and 6 months later was 201 $(p=0.0004), 180(p=0.0061)$ and $125 \mu \mathrm{m}(p=0.0018)$, respectively. In the non-vitrectomised group, the mean CMT before the second implant was $520 \pm 210 \mu \mathrm{m}$. Like in the vitrectomised group, this value was better than that at baseline but worse than that at 6 months. The CMT following the second implant in this group at months 1,2 and 3 was $296 \pm 85,318 \pm 116$ and $467 \pm 174 \mu \mathrm{m}$, respectively. The mean difference between the pre-treatment and post treatment CMT after months 1, 2 and 3 was 227, 205 and $56 \mu \mathrm{m}$, respectively. The difference was statistically significant only for the values at months $1(p=0.0190)$ and 3 $(p=0.0280)$ post implantation (Fig. 2e).

In the third round of treatment, the mean CMT before the final implant was $424 \pm 99 \mu \mathrm{m}$ in the vitrectomised group and $525 \pm 139 \mu \mathrm{m}$ in the non-vitrectomised group. In both 
groups, the CMT value pre-implantation was improved from baseline and worse than the CMT at 6 months. In the vitrectomised group, the mean CMT following the third implantation was $285 \pm 133 \mu \mathrm{m}$ at month $1,302 \pm 112 \mu \mathrm{m}$ at month 3 and $367 \pm 122 \mu \mathrm{m}$ at month 6 . The mean difference between the pre-implantation and post implantation CMT was $138 \mu \mathrm{m}$ at month $1,122 \mu \mathrm{m}$ at month 3 and $57 \mu \mathrm{m}$ at month 6. Again, the difference was statistically significant only for the values at months $1(p=0.0130)$ and 3 ( $p=$ 0.0018 , Fig. 2f). In the non-vitrectomised group, the mean CMT following the third implantation was $290 \pm 87 \mu \mathrm{m}$ at month $1,310 \pm 105 \mu \mathrm{m}$ at month 3 and $435 \pm 172 \mu \mathrm{m}$ at month 6 . In this groups, the mean difference between preimplantation and post implantation was 235, 214 and $90 \mu \mathrm{m}$, respectively, at months 1,2 and 3 after treatment; these differences were statistically significant $(p=0.0009$, $p=0.0013$ and $p=00081$, respectively; Fig. 2f).

The mean BCVA and CMT values were also improved from baseline after 13,15 and 18 months of treatment. In the vitrectomised group, the mean BCVA improvement was 4 lines (0.39 $\log$ MAR) after 13 and 15 months; after 18 months of treatment, the improvement was 2 lines $(0.22$ $\log \mathrm{MAR})$. In the non-vitrectomised group, the BCVA improved by 3 lines (0.28 logMAR), 2.5 lines (0.26 logMAR) and 1 line (0.14 $\log$ MAR), respectively, after 13,15 and 18 months of treatment (Table 1 ). The mean reduction in CMT in the vitrectomised group was $256 \mu \mathrm{m}$ (47\%), 239 $\mu \mathrm{m}(44 \%)$ and $175 \mu \mathrm{m}(32 \%)$, respectively, after 13,15 and 18 months of treatment. The mean reduction in CMT in the non-vitrectomised group was $350 \mu \mathrm{m}(54 \%), 329(51 \%)$ and $205 \mu \mathrm{m}(32 \%)$, respectively, after 13,15 and 18 months of treatment (Table 1). In conclusion, there was no statistically significant difference in BCVA or CMT between the two groups at any time point during the three rounds of treatment.

Finally, no serious systemic or ocular adverse events occurred in any of the patients who participated in this study. Most of the minor adverse events that occurred were related to the intraocular injection (Table 2). It is well known from previous studies that increased IOP is a common adverse event in patients receiving intraocular steroid treatment $[18,28]$. In our study, increased IOP (>20 mmHg) occurred in some patients in both groups. Five eyes $(50 \%)$ in the vitrectomised group and two (29\%) in the nonvitrectomised group developed increased IOP. Only a small percentage of our patients suffered from a clinically significant increase in IOP (>24 mmHg). Three eyes $(30 \%)$ in the vitrectomised group and $1(14 \%)$ in the nonvitrectomised group developed significantly increased IOP; in both groups, the increase in IOP occurred in months 1 and 3 post implantation and usually in the first two cycles of treatment (Table 2). Given that the increase in IOP was always transient, the increased IOP in these patients was subsequently controlled with IOP-lowering medication or observation. None of the patients needed laser or surgical intervention during the treatment period.

\section{Discussion}

In this study, we showed that the onset and duration of action and efficacy of the sustained-release dexamethasone (Ozurdex) implant were similar in vitrectomised and nonvitrectomised eyes with DMO during 18 months of followup. We found that the improvement in BCVA from preimplant values was statistically significant in both groups at months 1-3 after implantation in all three rounds of treatment. The peak improvement was observed 1 month after implantation. The CMT followed the same temporal pattern of improvement from pre-implantation values in both groups. From month 3 until month 6 after implantation, there was a regression in CMT and BCVA, but the values for both were still improved in both groups relative to baseline values in all rounds of treatment. Interestingly, the mean reduction in CMT after 13, 15 and 18 months of treatment was significantly reduced compared with baseline in the vitrectomised and non-vitrectomised groups. BCVA was also significantly improved from baseline in both groups, with the difference ranging from 1 to 4 lines. The above data show that the BCVA and CMT values were significantly improved related to baseline when implant was inserted at 6-monthly intervals.

The most important adverse event that occurred in our study was an increase in IOP. IOP-lowering medications were sufficient to control IOP in all cases. During the 18 months of continuous treatment with dexamethasone, the mean increase in IOP from baseline values was $2.1 \mathrm{mmHg}$ in the vitrectomised group and $1.7 \mathrm{mmHg}$ in the nonvitrectomised group (Table 2).

It is important to acknowledge the gradual deterioration in BCVA and CMT values from 3 months after dexamethasone implantation onwards in our study. The same activity pattern has also been noted by other researchers [18, 27, 29, 30]. However, the incidence of a clinically significant increase in IOP in our patients treated with the dexamethasone implant was low as in other similar studies [18, 27]. Overall the evidence appears to reinforce the discussion regarding the role of shorter treatment intervals [28, 31, 32], at least in patients at low risk for adverse events. These patients could retain a useful BCVA with minimal fluctuation during longterm treatment if re-treated earlier than 6 months. This possibility needs to be investigated further in the future. Our data are in agreement with the previous studies showing no difference in the efficacy of the dexamethasone (Ozurdex) implant according to whether the eye has undergone vitrectomy or not [27, 29, 33-35]. 
The main limitations of our study are the small number of patients, the lack of randomisation, and the retrospective design. Nevertheless, our study is the first to assess the efficacy and duration of action of the Ozurdex implant in vitrectomised and non-vitrectomised eyes that have been treated previously with anti-VEGF agents and triamcinolone for persistent DMO. Moreover, the majority of our patients who switched to the Ozurdex implant underwent more than 1 intravitreal implantation (the mean number of implantations was 2.5 in the vitrectomised group and 2.7 in the non-vitrectomised group). Studies that include larger numbers of patients in their vitrectomised and nonvitrectomised groups and with various pathologies (DMO, retinal vein occlusion, inflammatory $\mathrm{MO}$ ) are needed to obtain more information concerning the functional and anatomical efficacy of the dexamethasone implant in vitrectomised patients.

From previous studies, we know that the pharmacokinetics of triamcinolone, a corticosteroid used off-label to treat unresponsive DMO and retinal vein occlusions, are severely altered in vitrectomised eyes [23, 27]. It is also known from previous studies in animals and humans that the pharmacokinetics of anti-VEGF agents are altered in vitrectomised eyes, showing a faster clearance rate and a slower rate of improvement in CMT [30, 36, 37]. There has been some debate in recent years concerning the level of efficacy of anti-VEGF for the treatment of MO in vitrectomised eyes. As shown in our study, the long-acting therapeutic properties of the dexamethasone implant can make it a good therapeutic option for the treatment of persistent DMO in vitrectomised patients. Furthermore, the implant has a low risk of ocular side effects in patients who are pseudophakic, those who are not steroid responders, and those without glaucoma.

\section{Summary}

\section{What was known before}

- Increased clearance of intravitreal drugs in vitrectomised eyes.

- As a results shorter drug exposure time on retina tissue that decreases drugs efficacy.

- Both a-VEGF and triamcinolone (and many other drugs) show the previously described pharmacokinetic profile in vitrectomised eyes.

- CHAMPLAIN study analysed eyes that undergone vitrectomy in the past with difficult-to-treat DME.The study showed that single dexamethasone implantation improves vision as well as in central macular thickness (CMT), compared to baseline values.

- Other studies presented that Ozurdex has unaltered efficacy properties in vitrectomized eyes in comparison with non-vitrectomized eyes after a single injection (Eyes with DME).

\section{What this study adds}

- In the current research, the results support the assumption that dexamethasone intravitreal implant efficacy is not affected by previous vitrectomy surgeries in eyes with persistent DME.

- It is the first-time Ozurdex efficacy and temporal potency of action assayed in vitrectomised and nonvitrectomised eyes with persistent DME (no good response to a-VEGFs) that respond well to corticosteroids (previous triamcinolone treatment).

- Moreover, it is the first-time long-term follow-up characteristics (max 18 months) of Ozurdex treatment assayed in both groups.

- No difference in BCVA and CMT observed between vitrectomised and non-vitrectomised groups.

Acknowledgements We would like to acknowledge the help and support provided by our department staff members, Zambia Mirthianou, Sofia Sidiropoulou and Chrisoula Vlaxou; we greatly appreciate all the assistance provided.

\section{Compliance with ethical standards}

Conflict of interest The authors declare that they have no conflict of interest.

\section{References}

1. Das A, McGuire PG, Rangasamy S. Diabetic macular edema: pathophysiology and novel therapeutic targets. Ophthalmology. 2015;122:1375-94.

2. Tomkins-Netzer O, Ismetova F, Bar A, Seguin-Greenstein S, Kramer M, Lightman S. Functional outcome of macular edema in different retinal disorders. Prog Retin Eye Res. 2015;48:119-36.

3. Bringmann A, Reichenbach A, Wiedemann P. Pathomechanisms of cystoid macular edema. Ophthalmic Res. 2004;36:241-9.

4. Romano V, Angi M, Scotti F, del Grosso R, Romano D, Semeraro $\mathrm{F}$, et al. Inflammation and macular oedema after pars plana vitrectomy. Mediat Inflamm. 2013;2013:971758.

5. Lorenzi M. The polyol pathway as a mechanism for diabetic retinopathy: attractive, elusive, and resilient. Exp Diabetes Res. 2007;2007:61038.

6. Owen LA, Hartnett ME. Soluble mediators of diabetic macular edema: the diagnostic role of aqueous VEGF and cytokine levels in diabetic macular edema. Curr Diab Rep. 2013;13:476-80.

7. Funatsu H, Yamashita H, Ikeda T, Nakanishi Y, Kitano S, Hori S. Angiotensin II and vascular endothelial growth factor in the vitreous fluid of patients with diabetic macular edema and other retinal disorders. Am J Ophthalmol. 2002;133:537-43.

8. Funatsu H, Yamashita H, Ikeda T, Mimura T, Eguchi S, Hori S. Vitreous levels of interleukin- 6 and vascular endothelial growth factor are related to diabetic macular edema. Ophthalmology. 2003;110:1690-6. 
9. Boland EW. Nonspecific anti-inflammatory agents. Some notes on their practical application, especially in rheumatic disorders. Calif Med. 1964;100:145-55.

10. Bruno A, Cavallo-Perin P, Cassader M, Pagano G. Deflazacort vs prednisone. Effect on blood glucose control in insulin-treated diabetics. Arch Intern Med. 1987;147:679-80.

11. Stanbury RM, Graham EM. Systemic corticosteroid therapy-side effects and their management. Br J Ophthalmol. 1998;82:704-8.

12. Chen SD, Lochhead J, Patel CK, Frith P. Intravitreal triamcinolone acetonide for ischaemic macular oedema caused by branch retinal vein occlusion. Br J Ophthalmol. 2004;88:154-5.

13. Cheng CK, Berger AS, Pearson PA, Ashton P, Jaffe GJ. Intravitreal sustained-release dexamethasone device in the treatment of experimental uveitis. Invest Ophthalmol Vis Sci. 1995;36:442-53.

14. Diabetic Retinopathy Clinical Research N, Elman MJ, Qin H, Aiello LP, Beck, RW, Bressler NM, Ferris FL, et al. Intravitreal ranibizumab for diabetic macular edema with prompt versus deferred laser treatment: three-year randomized trial results. Ophthalmology. 2012;119:2312-8.

15. Bressler SB, Glassman AR, Almukhtar T, Bressler NM, Ferris FL, Googe JM Jr, et al. Five-year outcomes of ranibizumab with prompt or deferred laser versus laser or triamcinolone plus deferred ranibizumab for diabetic macular edema. Am J Ophthalmol. 2016;164:57-68.

16. Diabetic Retinopathy Clinical Research N, Elman MJ, Aiello LP, Beck RW, Bressler NM, Bressler SB, Edwards AR, et al. Randomized trial evaluating ranibizumab plus prompt or deferred laser or triamcinolone plus prompt laser for diabetic macular edema. Ophthalmology. 2010;117:1064-77.e35.

17. Beer PM, Bakri SJ, Singh RJ, Liu W, Peters GB 3rd, Miller M. Intraocular concentration and pharmacokinetics of triamcinolone acetonide after a single intravitreal injection. Ophthalmology. 2003;110:681-6.

18. Boyer DS, Yoon YH, Belfort R Jr, Bandello F, Maturi RK, Augustin AJ, et al. Three-year, randomized, sham-controlled trial of dexamethasone intravitreal implant in patients with diabetic macular edema. Ophthalmology. 2014;121:1904-14.

19. Campochiaro PA, Brown DM, Pearson A, Ciulla T, Boyer D, Holz FG, et al. Long-term benefit of sustained-delivery fluocinolone acetonide vitreous inserts for diabetic macular edema. Ophthalmology. 2011;118:626-35.e2.

20. Haller JA, Bandello F, Belfort R Jr, Blumenkranz MS, Gillies M, Heier J, et al. Randomized, sham-controlled trial of dexamethasone intravitreal implant in patients with macular edema due to retinal vein occlusion. Ophthalmology. 2010;117:1134-46.e3.

21. Thakur A, Kadam R, Kompella UB. Trabecular meshwork and lens partitioning of corticosteroids: implications for elevated intraocular pressure and cataracts. Arch Ophthalmol. 2011;129:914-20.

22. Lee SS, Ghosn C, Yu Z, Zacharias LC, Kao H, Lanni C, et al. Vitreous VEGF clearance is increased after vitrectomy. Invest Ophthalmol Vis Sci. 2010;51:2135-8.

23. Chin HS, Park TS, Moon YS, Oh JH. Difference in clearance of intravitreal triamcinolone acetonide between vitrectomized and nonvitrectomized eyes. Retina. 2005;25:556-60.
24. Schindler RH, Chandler D, Thresher R, Machemer R. The clearance of intravitreal triamcinolone acetonide. Am J Ophthalmol. 1982;93:415-7.

25. Doft BH, Weiskopf J, Nilsson-Ehle I, Wingard LB Jr Amphotericin clearance in vitrectomized versus nonvitrectomized eyes. Ophthalmology. 1985;92:1601-5.

26. Jarus G, Blumenkranz M, Hernandez E, Sossi N. Clearance of intravitreal fluorouracil. Normal and aphakic vitrectomized eyes. Ophthalmology. 1985;92:91-6.

27. Boyer DS, Faber D, Gupta, S, Patel SS, Tabandeh H, Li XY, et al. Dexamethasone intravitreal implant for treatment of diabetic macular edema in vitrectomized patients. Retina. 2011;31:915-23.

28. Scaramuzzi M, Querques G, Spina CL, Lattanzio R, Bandello F,, et al. Repeated intravitreal dexamethasone implant (Ozurdex) for diabetic macular edema. Retina. 2015;35:1216-22.

29. Medeiros MD, Alkabes M, Navarro R, Garcia-Arumi J, Mateo C, Corcostegui B. Dexamethasone intravitreal implant in vitrectomized versus nonvitrectomized eyes for treatment of patients with persistent diabetic macular edema. J Ocul Pharmacol Ther. 2014;30:709-16.

30. Bressler SB, Melia M, Glassman AR, Almukhtar T, Jampol LM, Shami M, et al. Ranibizumab plus prompt or deferred laser for diabetic macular edema in eyes with vitrectomy before antivascular endothelial growth factor therapy. Retina. 2015;35:2516-28.

31. Ramu J, Yang Y, Menon G, Bailey C, Narendran N, Bunce C, et al. A randomized clinical trial comparing fixed vs pro-re-nata dosing of Ozurdex in refractory diabetic macular oedema (OZDRY study). Eye. 2015;29:1603-12.

32. Arikan Yorgun M, Toklu Y, Mutlu M. Comparison of early dexamethasone retreatment versus standard dexamethasone regimen combined with PRN ranibizumab in diabetic macular edema. Int Ophthalmol. 2017;1:185-96.

33. Reibaldi M, Russo A, Zagari M, Toro M, Grande De V, Cifalino $\mathrm{V}$, et al. Resolution of persistent cystoid macular edema due to central retinal vein occlusion in a vitrectomized eye following intravitreal implant of dexamethasone $0.7 \mathrm{mg}$. Case Rep Ophthalmol. 2012;3:30-4.

34. Adan A, Pelegrin L, Rey A, Llorenc V, Mesquida M, Molins, B, et al. Dexamethasone intravitreal implant for treatment of uveitic persistent cystoid macular edema in vitrectomized patients. Retina. 2013;33:1435-40.

35. Chang-Lin JE, Burke JA, Peng Q, Lin T, Orilla WC, Ghosn CR, et al. Pharmacokinetics of a sustained-release dexamethasone intravitreal implant in vitrectomized and nonvitrectomized eyes. Invest Ophthalmol Vis Sci. 2011;52:4605-9.

36. Christoforidis JB, Williams MM, Wang J, Jiang A, Pratt C, AbdelRasoul M, et al. Anatomic and pharmacokinetic properties of intravitreal bevacizumab and ranibizumab after vitrectomy and lensectomy. Retina. 2013;33:946-52.

37. Ahn SJ, Ahn J, Park S, Kim H, Hwang DJ, Park JH, et al. Intraocular pharmacokinetics of ranibizumab in vitrectomized versus nonvitrectomized eyes. Invest Ophthalmol Vis Sci. 2014;55:567-73 